\title{
Low Confinement Loss Photonic Crystal Fiber (PCF) With Flat Dispersion over C-Band
}

\author{
Shiju. C. Chacko \\ Assistant Professor \\ Department of Electronics \& \\ Communication Engineering \\ Hindustan University
}

\author{
Jeena Maria Cherian \\ Assistant Professor \\ Department of Electronics \& \\ Communication Engineering \\ Hindustan University
}

\author{
Sunilkumar K \\ Assistant Professor \\ Department of Electronics \& \\ Communication Engineering \\ Hindustan University
}

\begin{abstract}
A new structure of low confinement loss with flat dispersion PCF over C-band is proposed and analysed by full vector finite element method. A solid core PCF has been designed with circular air-holes of different diameters and the background index are calculated from Sellmeier equation. From the results it is confirmed that the proposed design finds application in communication with its extremely low confinement loss and flat dispersion over the $\mathrm{C}$ - band. Within the wavelength band $1.5 \mu \mathrm{m}$ to $1.6 \mu \mathrm{m}$ the fast axis mode vanishes leaving single polarization.
\end{abstract}

\section{Keywords}

Photonic Crystal Fiber (PCF), birefringence, confinement loss, dispersion, Finite element Method (FEM)

\section{INTRODUCTION}

The use of optical fiber in communication has revolutionized the telecommunication industry in the way signal is transmitted. Photonic Crystal Fiber (PCF), with long periodic arrangement of air holes running along the longitudinal direction, shows many interesting characteristics unachievable by conventional optical fiber. By tuning the air holes or with special transverse structure design, the dispersion can be tailored to compliment the weakness of optical systems [1].

In contrast to the conventional fibers, layers of microstructured fibers may consist of several different materials and contain photonic crystal-like microstructure. The size of microstructure elements is of the order of radiation wavelength propagating over this fiber. Main peculiarity of such fibers is strong dependence of its optical properties on the geometry of the microstructure. It gives possibility to design the fiber with any possible characteristics by manipulation with geometry only.

The two different guiding mechanisms in photonic crystal fibers are index guiding, solid-core PCF and light propagation by the hollow-core photonic band gap (PBG) effect at the operating wavelength with a low index core region. Solid core PCFs work on the principle of modified total internal reflection while the guiding mechanism in hollow PCFs is based on band gaps. The main parameter of a PCF is its effective index of refraction $\mathrm{n}_{\text {eff }}$ whose real part contains the dispersion information $\mathrm{D}$, whereas the imaginary part allows the calculation of the losses $\mathrm{L}$.

PCF's find application in ever widening areas of science and technology like high power energy transmission, fiber LASER's, intra-fiber LASER's.

The confinement loss and dispersion properties are discussed using full-vectorial finite element method. By implementing structures with dissimilar air-hole diameter, varying spacing between air holes and the number of air holes in the cladding, the confinement loss can be conveniently engineered.

This paper presents a new cladding design with low confinement loss with flat dispersion over the C- band which offers the usage of compatible systems such as erbium doped fiber amplifier EDFA and LASER sources. The finite element method (FEM) effectively eliminates the differential part or convert it into ordinary differenatial form which are then integrated by standered techniques like Euler's method, Runge Kutta etc.

\section{PROPERTIES}

A huge variety of air hole arrangements are possible with a $\mathrm{PCF}$, due to which the design offers a wide possibility to control the refractive contrast between the core and the cladding and as a consequence, novel and unique optical properties can be achieved. The tendency of different wavelengths to travel at different velocities is a crucial factor in telecommunication system design

The leakage losses or confinement losses associated with the PCF's discriminates one mode from the others; hence, one can have structures with wide effectively-single-mode operation wavelength range [2] or non-polarization-degenerate effectively-single-mode operation $[3,4]$. Therefore, the ability to calculate both the real and imaginary part of the modal indices is important.

$$
L=\frac{40 \pi}{\ln (10) \lambda} \operatorname{Im}(n \text { eff })
$$

in $\mathrm{dB} / \mathrm{m}$, where the Im stands for the imaginary part.

A single-mode fiber is not truly single mode because it can support two degenerate modes that are polarized in two orthogonal directions. Under ideal conditions, a mode excited with its polarization in the $\mathrm{x}$ direction would not couple to the mode with the orthogonal y-polarization state. In real fibers, small departures from cylindrical symmetry because of random variations in core shape and stress-induced anisotropy result in a mixing of the two polarization states by breaking the mode degeneracy. Mathematically, the mode propagation constant becomes slightly different for the modes polarized in the $\mathrm{x}$ and $\mathrm{y}$ directions. This property is referred to as modal birefringence. The strength of modal birefringence is defined as [5]

$$
\beta_{m}=n_{y}-n_{x}
$$

If an input pulse excites the two polarization components, the two components travel along the fiber at different speeds because of their different group velocities. The pulse becomes broader at the output end because group velocities change randomly in response to random changes in fiber 
birefringence. This phenomenon, referred to as polarizationmode dispersion (PMD).

In PCF's, the dispersion can be controlled and tailored with unprecedented freedom, due to the high refractive index difference between Silica and the air holes. Also the flexibility of changing the air hole sizes and patterns offers broader range of dispersion behaviours.

$$
D=-\left(\frac{\lambda}{C}\right) \frac{\partial^{2}}{\partial \lambda^{2}} \operatorname{Re}(n \text { eff })
$$

\section{B. ANALYSIS METHOD}

From Maxwell's equation, the basic equation for FEM analysis is given as

$$
\nabla \mathrm{x}\left(\mu_{r}^{-1} \nabla \mathrm{xE}\right)-k_{0}^{2} \epsilon_{r} E=0
$$

where $\mathrm{k}_{0}$ is the free space wavenumber, $\mathrm{E}$ denotes the electric field and $\varepsilon_{\mathrm{r}} \mu_{\mathrm{r}}$ are the relative dielectric permittivity and magnetic permeability respectively.

When applying a full-vector FEM to PCFs, a curvilinear hybrid edge/nodal element [7] is very useful for avoiding spurious solutions and for accurately modelling curved boundaries of air holes. Dividing the fiber cross section into a number of curvilinear hybrid elements, from (4) it is possible to obtain the following eigenvalue equation

$$
[K]\{E\}=k_{0}^{2} n_{e f f}^{2}[M]\{E\}
$$

where $[\mathrm{K}]$ and $[\mathrm{M}]$ are the finite element matrices, $\{\mathrm{E}\}$ is the discretized electric field vector consisting of the edge and nodal variables, $\mathrm{n}_{\mathrm{eff}}$ is the effective index, and anisotropic PMLs are incorporated as an absorbing boundary condition to evaluate confinement losses.

\section{FLAT DISPERSION-LOW CONFINEMENT LOSS PCF}

Consider the PCF design as shown in Fig. 1, where D is the hole pitch (center-to-center distance between the holes) $d_{1}$ and $\mathrm{d}_{2}$ are the diameters of the small and large air holes, respectively. The design principle is to have the cut-off wavelengths of the fundamental modes polarized along the slow axis (slow-axis mode) and the fast axis (fast-axis mode) longer and shorter than the operating wavelength, respectively. The cut-off of either polarization occurs when its effective refractive index becomes equal to the cladding effective index of the fundamental space-filling mode (FSM) [8].

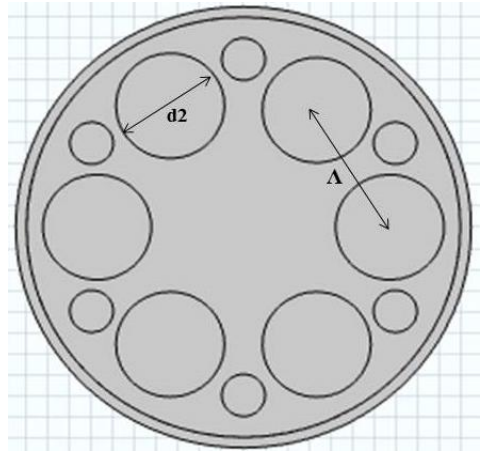

Fig. 1. PCF Design

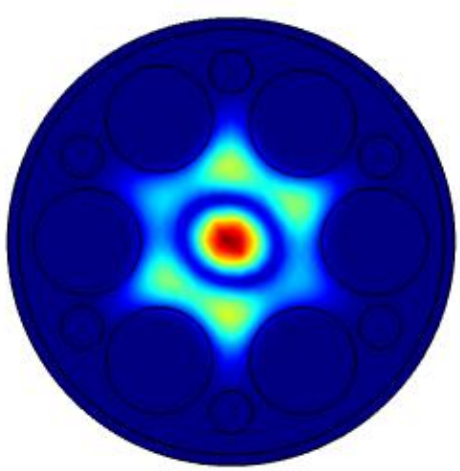

Fig. 2 Intensity Profile of slow axis mode

Fig. 3 shows the effective refractive index fast axis and slow axis as a function of wavelength. The background index of Silica is calculated through Sellmeier equation. The fast axis mode is unguided in the wavelength range over $\mathrm{C}$-band so a single polarization operation is achieved. It is found that the confinement loss is very low over the communication band and remains almost constant for this the same spectrum.

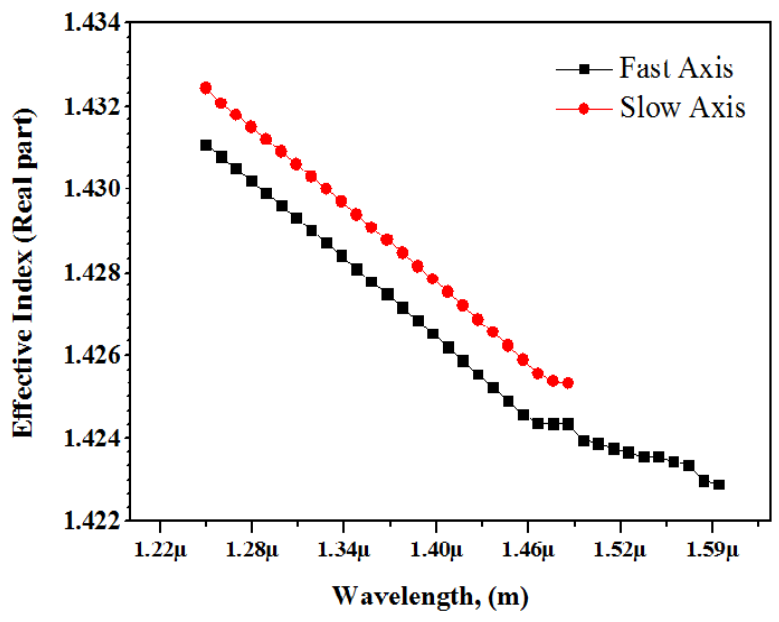

Fig. 3. Effective Refractive Index, Real part

Fig. 4 shows the wavelength dependence of the confinement loss. In PCF;s with an infinite number of air holes confinement loss do not occur, In fabricated PCF's however the number of air holes in the cladding is finite and the modes of such fibers are leaky.

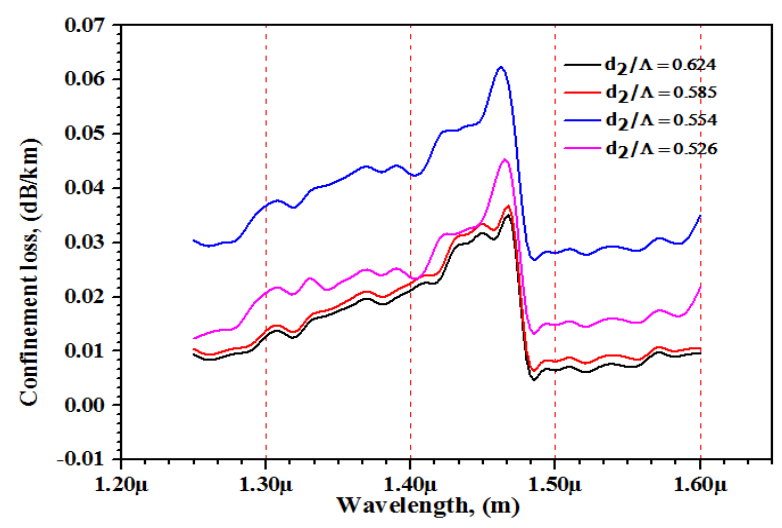

Fig. 4. Confinement Loss 
Fig.5 shows dispersion characteristics as function of wavelength and various for $\mathrm{d} / \mathrm{D}$ values. It has been observed that the dispersion remains low over the wavelength range $1500 \mathrm{~nm}$ to $1600 \mathrm{~nm}$ which is used for optical communication application.

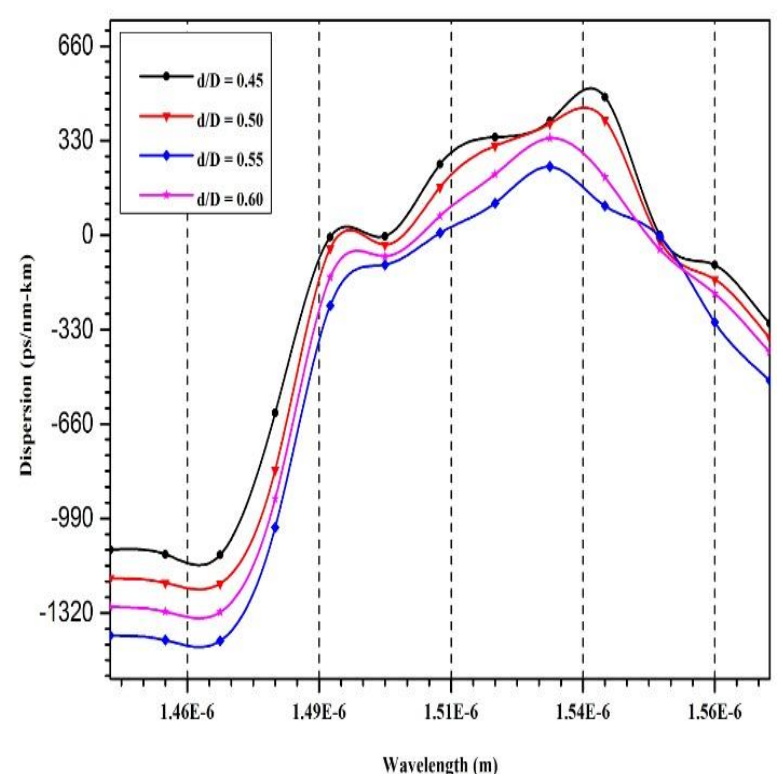

Fig. 5. Dispersion Characteristics for various $d / D$ values

\section{CONCLUSION}

A new structure of PCF with low confinement loss and flat dispersion is proposed and numerically analyzed by using full vector finite element method. The novel design offers flat dispersion and low confinement loss over the C- band with single polarization. The new design offers flat dispersion over 1.49 to 1.54 micrometer band. Also it has been shown that the confinement loss has been sharply dropped down to a very low value at the proffered communication wavelength for this design. The dispersion can be made zero or closely zero with novel cladding design but with a trade off in confinement loss.

\section{REFERENCES}

[1] D. C. Tee et. al. 2013. Photonic Crystal Fiber in Photonic Crystal Fiber for residual Dispersion Compensation Over $\mathrm{E}+\mathrm{S}+\mathrm{C}+\mathrm{L}+\mathrm{U}$ Wavelength Bands, IEEE Photonics Journal.

[2] T.A. Birks, J.C. Knight, and P.S.J. Russell, "Endlessly single- mode photonic crystal fiber," Opt. Lett. 22, 961-963 (1997).

[3] I.M. Basset and A. Argyros, "Elimination of polarization degeneracy in round waveguides," Opt. Express 10,1342-1346 (2002).

[4] A. Argyros, N. Issa, I. Basset, and M. van Eijkelenborg, "Microstructured optical fiber for single-polarization air guidance," Opt. Lett. 29, 2022 (2004).Tavel, P. 2007 Modeling and Simulation Design. AK Peters Ltd.

[5] I. P. Kaminow, IEEE J. Quantum Electron. QE-17, 15 (1981)

[6] G. P. Agrawal, "Nonlinear Fiber Optics", Academic Press, $3^{\text {rd }}$ edition, 2003

[7] M. Koshiba and Y. Tsuji, "Curvilinear hybrid edge/nodal elements with triangular shape for guided-wave problems," J. Lightwave Technol., vol.18, pp. 737-743, May 2000.

[8] Y.T. Yu, M.F. Lau, "A comparison of MC/DC MUMCUT and several other coverage criteria for logical decisions", Journal of Systems and Software, 2005 , in press.

[9] T. A. Birks, J. C. Knight, and P. S. J.P. St. J. Russell, "Endlessly single-mode photonic crystal fiber," Opt. Lett., vol. 22, pp. 961-963,July 1997 